\title{
INSERCIÓN DEL SISTEMA DE GESTIÓN AMBIENTAL EN EL PROGRAMA DE INGENIERÍA MECÁNICA DE LA UNIVERSIDAD FRANCISCO DE PAULA SANTANDER OCAÑA
}

\section{INSERTION OF THE ENVIRONMENTAL MANAGEMENT SYSTEM IN THE MECHANICAL ENGINEERING PROGRAM OF THE FRANCISCO DE PAULA SANTANDER OCAÑA UNIVERSITY}

\author{
Esp. Malka Irina Cabellos Martínez*, Esp. Jhon Arévalo Toscano** \\ * Universidad Francisco de Paula Santander Ocaña, Facultad de Ingenierías, Grupo \\ de Investigación INGAP \\ Sede Algodonal, Ocaña, Norte de Santander, Colombia. \\ 0975690088 ext 210 \\ E-mail: micabellosm@ufpso.edu.co \\ ** Universidad Francisco de Paula Santander Ocaña, Facultad de Ingenierías, Grupo \\ de Investigación INGAP \\ Sede Algodonal, Ocaña, Norte de Santander, Colombia. \\ 0975690088 ext 210 \\ E-mail: jarevalot@ufpso.edu.co
}

Resumen: El propósito de este trabajo consiste en realizar una propuesta para la inserción del Sistema de Gestión Ambiental en el programa de Ingeniería Mecánica de La Universidad Francisco de Paula Santander Ocaña, con el fin de identificar los criterios y determinar los parámetros definidos para la aplicación de la responsabilidad social universitaria en el aspecto ambiental desde el plan de estudios. Para tal fin, se implementa una investigación con un enfoque cuantitativo, de tipo descriptivo que permitirá caracterizar para el programa académico a través de analizar la variable ambiental de la responsabilidad social universitaria para obtener una propuesta del Sistema de Gestión Ambiental (SIGA) en el Programa de Ingeniería Mecánica de la Universidad Francisco de Paula Santander Ocaña enfocado al uso eficiente de la energía y cuidado del medio ambiente.

El presente trabajo incluye el análisis de la estructura organizada, responsabilidades, planificación de las actividades, prácticas, procedimientos, procesos y los recursos para desarrollar, implantar y mantener al día la política medioambiental de la Universidad Francisco de Paula Santander Ocaña a través del programa de Ingeniería Mecánica. En este sentido, se esperan contar con una caracterización que permita validar el Sistema de Gestión Ambiental, y así consolidar como los Sistemas de Gestión Ambiental deben ser apropiados a la naturaleza e impactos medioambientales de sus actividades, productos y servicios; para que el programa de Ingeniería Mecánica tenga un compromiso de mejora continua y de prevención de la contaminación en un marco adecuado para el establecimiento y revisión de los objetivos y metas medioambientales de la institución.

Palabras clave: Sistema; Gestión Ambiental; Responsabilidad Social; Programa Académico. 


\begin{abstract}
Our journal The purpose of this research consists of a proposal of insertion of the Environmental Management System in the Mechanical Engineering Program from the University Francisco de Paula Santander Ocaña, with the aim of finding out the judgments and establishing the definite criteria for the correct application of social university responsibility in the environment facet from the engineering career office. In addition, it is implemented a descriptive quantitative research, which will let characterize the Engineering Program throughout the analysis of the environmental variable of social university responsibility to obtain a proposal of the Environmental Management System (EMS) in the Mechanical Engineering Program from the University Francisco de Paula Santander Ocaña, being focused on the efficient consumption of electricity and the care of the environment.

This research includes the analysis of the organized infrastructure, responsibilities, activity plans, practices, procedures, processes and the resources of developing, implementing and updating the environment politics from the University Francisco de Paula Santander Ocaña throughout the Mechanical Engineering Program. Moreover, it is hoped to have a characterization that allows validating the Environmental Management System, and in that way to consolidate how the Environmental Management Systems must be appropriate for nature and environment impacts in their activities, products and services; finally the Mechanical Engineering Program can have a commitment of a continuous improvement and polluting prevention in an adequate context for establishing and checking the university environmental objectives and goals.
\end{abstract}

Keywords: System; Environmental Management, Social Responsibility, Academic Program.

\section{INTRODUCCIÓN}

La presente investigación se desarrolló en el contexto del programa de Ingeniería mecánica, al situarse en el Proyecto Educativo del Programa (PEP) el propósito de formación integral en los estudiantes de este plan de estudios con una búsqueda constante en que se vea reflejado en los futuros profesionales la responsabilidad de construir una sociedad mejor, inmersa en una región que busca que la sostenibilidad, el uso eficiente de la energía y el cuidado del medio ambiente sea una nueva forma de vida (Puerto Suarez \& Lopez Jimenez, 2014).

De acuerdo con Hernández y Saldarriaga (2008, p. 238) "La principal razón para hablar de Responsabilidad Social es porque más allá de ser una estrategia es el distintivo de calidad de cualquier organización que busque la transformación social para lograr el desarrollo"(Hernandez \& Saldarriga, 2009). Es por ello que el Ingeniero Mecánico de la UFPS Ocaña establece la diferencia al estar capacitado para solucionar problemas en contexto.

Así mismo, se busca fortalecer a través de la Responsabilidad Social del plan de estudios los planes y programas con los que se cuenta para que los estudiantes reciban en su formación la importancia y las competencias por el uso eficiente de la energía y el cuidado del medio ambiente. El alcance del proyecto se define para la ciudad de Ocaña Norte de Santander, específicamente en este programa académico adscrito a la Facultad de Ingenierías.

En los programas académicos se observan indicadores reales del profesional integral que se ha formado en la institución. Es por ello que en el perfil del egresado del ingeniero Mecánico UFPSO se espera encontrar un ser integral solucionador de problemas en contexto, responsable con el medio ambiente y con lineamientos claros acerca del desarrollo sostenible. Este propósito, encontraría algunos inconvenientes considerando que las políticas institucionales de medio ambiente y uso eficiente de los recursos, a pesar de estar proyectadas en la docencia, investigación y extensión no se ven reflejadas en el actuar de los programas académicos, de manera específica en el programa de Ingeniería Mecánica no existe un documento o procedimiento en donde se establezca la política del programa académico desde el eje ambiental. Por tal razón, el presente trabajo de investigación busca evidenciar las debilidades en el proceso de formación del enfoque del ingeniero mecánico con Responsabilidad Social desde la mirada de una variable ambiental; así de esta 
manera puedan ser trazados planes, programas y estrategias en aras de fortalecer a través del currículo la formación de los ingenieros mecánicos que brinden a la sociedad soluciones fundamentadas en el desarrollo sostenible.

Otro indicador importante que se hace visible es poder integrar las TIC de forma transversal en las funciones propias de la organización a través de la implementación de la Responsabilidad Social Universitaria en el programa de Ingeniería Mecánica analizando en el desarrollo curricular de formación en los diferentes actores del proceso docentes y estudiantes (Bonfante \& Castillo, 2014).

Se plantea una sensibilización en docentes del programa de Ingeniería Mecánica a reflexionar en la actualización del modelo micro curricular con el uso de herramientas computacionales que logren fortalecer estrategias de aprendizaje encaminadas al uso de software que permita encaminar a los estudiantes a analizar como logran ser protagonistas en la disminución del impacto ambiental (Rodríguez, Pinto, \& Cárdenas, 2012).

\section{DESARROLLO}

El desconocimiento del Sistema de Gestión Ambiental en un porcentaje de estudiantes y profesores en el programa de Ingeniería Mecánica evidencia algunas debilidades del mismo, que permiten formular estrategias a través de mecanismos como medición, evaluación y verificación encaminadas al fortalecimiento de la estructura curricular en el plan de estudios a través del Sistema Integrado de Gestión Ambiental de la Institución que permiten que se puedan formular estrategias que a través de mecanismos tales como medición, evaluación y verificación encaminen la estructura curricular en el plan de estudios a que pueda fortalecerse a través del Sistema Integrado de Gestión (SIGA) con herramientas de sensibilización, procedimientos, programas para que sean implementados y logren permear para que se alcance una cultura en los estudiantes y docentes que cumplan con la política ambiental de la Universidad Francisco de Paula Santander Ocaña. Por lo anterior, los mecanismos de implementación del uso de los recursos y desarrollo sostenible se verán reflejados desde la extensión del programa de Ingeniería Mecánica.

El plan de estudios no cuenta con planes y programas que permitan la inclusión de la Gestión Ambiental en el programa de Ingeniería Mecánica. No se observa en la malla curricular la trazabilidad de la variable ambiental en electivas y/o asignaturas propias del programa de formación en donde se encuentren identificados los resultados e indicadores en el cumplimiento de los Micro currículos de la casa de estudios que garanticen la variable ambiental como eje transformador de la formación de los ingenieros.

Se requiere en el programa de Ingeniería Mecánica de la U F P S Ocaña contar con indicadores en investigación, docencia y extensión que logren medir y verificar los diferentes planes y programas encaminados al uso eficiente de la energía y el cuidado del medio ambiente inmersos en el currículo y serán evidenciados a través de los estudiantes, docentes y los egresados que permiten la validación de la formación de los ingenieros.

En el marco de la Autoevaluación Institucional de la Universidad Francisco de Paula Santander Ocaña se han establecido unos lineamientos de control de la gestión y desde lo académico permite a partir de las acciones de mejora de los directivos, profesores, estudiantes, egresados, funcionarios, servidores y órganos gubernamentales se definan planes de mejoramiento que trazan la ruta para que desde cada uno de los procesos se le apueste al cumplimiento de los objetivos institucionales. El presente trabajo de investigación permite que el programa de Ingeniería Mecánica busque el cumplimiento de la Política Integral de la Universidad, la cual en su eje Ambiental está enfocada al medio ambiente y al uso eficiente de los recursos le apueste al cumplimiento de la Política Integral de la Universidad, la cual en su eje Ambiental le apuesta como institución a cuidar el medio ambiente y apostarle al uso eficiente de los recursos.

La pertinencia de la Educación Superior se evalúa en función de lo que la sociedad espera de las instituciones comparadas con lo que en realidad muestran sus indicadores. Por tal razón, es fundamental contar en las instituciones de educación superior con normas, políticas, planes y programas que logren permear en los planes de estudio para que se vean reflejados en la formación de los estudiantes, en este caso de los ingenieros mecánicos (Herrera, 2008).

El programa de Ingeniería Mecánica en Ocaña Norte de Santander, comparte el denominado " Modo 2, de Gibbons (1997) acerca de generar conocimiento útil socialmente en contextos específicos de aplicación " (Herrera, 2008). 
Lo anterior, considerando que el sector productivo en donde se ubican los egresados del programa aporta al desarrollo humano sostenible en nuestra región. Es así como el desarrollo del presente trabajo de investigación permitirá a la institución hablar de Responsabilidad Social Universitaria a través del programa de Ingeniería Mecánica que busca en la formación de los estudiantes un bienestar integral y un desarrollo humano sostenible y sustentable.

Se orienta la presente investigación a partir de los siguientes propósitos:

Caracterizar el programa de Ingeniería Mecánica de la Universidad Francisco de Paula Santander Ocaña en cuanto a la misión, el Proyecto Educativo y objetivos del Programa.

Determinar elementos de inserción en cuanto al Sistema de Gestión Ambiental para el programa de Ingeniería Mecánica de La Universidad Francisco De Paula Santander Ocaña.

Diseñar una propuesta de inserción del Sistema de Gestión Ambiental en el programa de Ingeniería Mecánica de La Universidad Francisco De Paula Santander Ocaña

Este proyecto de investigación se desarrolla durante el segundo semestre del año 2016, en el programa de Ingeniería Mecánica adscrito a la Facultad de Ingenierías de la Universidad Francisco de Paula Santander Ocaña, que se ubica en la región del Catatumbo en el departamento Norte de Santander. El programa de ingeniería mecánica de la UFPSO, nace del compromiso de la universidad por cumplir con su deber de formar profesionales que se sitúen en un contexto globalizado y puedan desempeñarse como agentes generadores de cambio social. Todo esto se fundamenta en los ideales de la institución, los cuales son satisfacer las necesidades de la región, para la formación profesional de ingenieros mecánicos en la provincia de Ocaña y los departamentos de Cesar, Bolívar, Guajira y en menor proporción Magdalena, Atlántico y los demás del país.

El programa de Ingeniería Mecánica consciente de su proceso de formación reconoce en la institución el Sistema de Gestión Ambiental (SIGA) dentro de la Política Integral de la Institución, pero se hace necesario conocer si este sistema ha logrado permear en el actuar de los docentes, estudiantes y administrativos del plan de estudios. Es por ello, que el presente trabajo permite analizar el nivel de articulación del SIGA y el plan de estudios de Ingeniería Mecánica.

\section{METODOLOGÍA}

Para la ejecución de los objetivos planteados se define una investigación con un enfoque cuantitativo y de tipo descriptivo; de acuerdo a lo planteado por McMillan y Scumacher (2005) en donde se dará respuesta a preguntas que permitan medir el nivel de apropiación de la responsabilidad social universitaria desde su eje ambiental en el plan de estudios de Ingeniería Mecánica. Por tanto, el trabajo es NO experimental considerando que no se manipulan ninguna de las variables.

El trabajo de campo de la presente investigación busca Identificar la inclusión del Sistema de Gestión Ambiental (SIGA) en el programa de Ingeniería Mecánica de la Facultad de Ingenierías de la Universidad Francisco de Paula Santander Ocaña enfocado al uso eficiente de la energía y cuidado del medio ambiente, para ello se aplica un instrumento de medición que permite verificar y controlar la articulación del SIGA en un plan de estudios específico.

Para la realización del diagnóstico del área de mejora del Sistema de Gestión Ambiental (SIGA) y su inserción en el Programa de Ingeniería Mecánica de la Universidad Francisco de Paula Santander Ocaña se tendrá como parámetro de seguimiento en el trabajo de campo los resultados de la encuesta aplicada a docentes y estudiantes.

A través de los instrumentos de medición se identifican las variables de estudio definidas a partir del nivel de la inserción que se tiene entre el SIGA y el programa de Ingeniería Mecánica de la Facultad de Ingenierías de la Universidad Francisco de Paula Santander Ocaña a través de la Responsabilidad enfocado al uso eficiente de la energía y cuidado del medio ambiente, esto con el fin que se determine el área de mejora. Es por ello, que se plantean como variables en el presente trabajo, la planeación de actividades que apoyan la protección del medio ambiente y la prevención de la contaminación y el nivel de inclusión de los procedimientos del Sistema de Gestión Ambiental para el cumplimiento de la política ambiental de la Universidad Francisco de Paula Santander Ocaña.

El programa de Ingeniería Mecánica cuenta con estudiantes de los diferentes municipios de los departamentos de sur del cesar, la guajira. Es así 
como se tienen 500 estudiantes en el segundo semestre de 2016 que tienen edades que oscilan entre los 16 y 28 años de edad. A partir de la población total en el plan de estudios, se obtiene para la muestra un valor de 218 estudiantes.

Por otro lado, la segunda población de estudio son los docentes, los cuales se clasifican en: profesores de planta, cátedra y ocasionales. Así mismo re realiza la selección al azar de 10 personas de este grupo como muestra de carácter aleatorio.

La recolección de la información se plantea a partir de una encuesta donde se evalúa en estudiantes y docentes del programa de Ingeniería Mecánica la percepción que tienen sobre algunos elementos que componen la Responsabilidad Social Universitaria frente a lo que estipula la Universidad Francisco de Paula Santander Ocaña en su SIGA a partir de información primaria. Como parte del diagnóstico se hace una revisión documental con información secundaria en donde se revisan las políticas institucionales de la Universidad Francisco de Paula Santander Ocaña tales como, Proyecto Educativo Institucional, Proyecto Educativo del Programa, Planes de Mejoramiento del programa de Ingeniería Mecánica, Política Integral de la U F P S Ocaña, Revisión documental del Sistema de Gestión Ambiental (SIGA) (Sánchez Ortiz, 2015).

Se realizó una investigación descriptiva, por eso el uso de la encuesta, con el fin de reseñar las características del fenómeno a partir de la muestra representativa de la comunidad académica (profesores y estudiantes); se obtuvieron 218 respuestas por parte de los estudiantes matriculados a partir del tercer semestre de Ingeniería Mecánica. Por su parte, con los docentes se obtuvieron 10 respuestas con profesores bajo la modalidad de catedrático o docente de tiempo completo.

\section{RESULTADOS}

A partir de la revisión documental de los registros institucionales para identificar los elementos de RSU con los que cuenta el programa de Ingeniería Mecánica en la Facultad de Ingenierías de la UFPS Ocaña con el fin de analizar el nivel de inserción que se tienen entre el SIGA y el plan de estudios de Ingeniería Mecánica en lo referente al eje ambiental enfocados al uso eficiente de la energía y cuidado del medio ambiente.

Con base al análisis del SIGA se logra evidenciar en su política ambiental un compromiso con las actividades de los docentes y estudiantes pero no se encuentran en el perfil del egresado del programa de Ingeniería Mecánica que se oriente su formación integral y el ejercicio de su profesión a lo expresado por François Vallaeys, (2008) para el cual, asumir la Responsabilidad Social Universitaria significa llevar a la institución a replantear el perfil de su egresado universitario (Vallaeys, De La cruz, \& Sasia, 2009). Lo cual permite que los profesionales que abordan las diferentes problemáticas en la sociedad cuenten con herramientas propositivas que desarrollen soluciones innovadoras que minimicen el impacto en el medio ambiente (G, Serrano, \& G., 2012).

De acuerdo a lo planteado por Mesa \& Barrera, (2012) desarrollar propuestas educativas innovadoras permite lograr en los estudiantes un aprendizaje significativo desde una experiencia educativa en torno a un tema específico a través de la comprensión de los fenómenos involucrados permitiendo que el estudiante pueda fundamentar sus argumentos a partir de los conceptos desarrollados en el aula.

Mejorar la calidad de la educación significa reflexionar en torno a las prácticas implementadas en el aula, lo cual significa que puedan ser orientados los contenidos y metodologías desarrolladas en el aula a un aprendizaje centrado en el alumno ( $\mathrm{G}$ et al., 2012). Esto encamina la reflexión, a repensar las competencias que son plasmadas en la formación profesional para el programa de Ingeniería Mecánica de la UFPS Ocaña.

De manera adicional se logra evidenciar en las competencias descritas desde el proyecto educativo del programa (PEP) para el plan de estudios de ingeniería mecánica los elementos de valoración de las competencias en cada estudiante a lo largo de las asignaturas establecidas en la malla curricular (Espinel Blanco, 2012), así como se presenta en la tabla 1 los desempeños académicos a fortalecer en el proceso de formación.

Tabla 1: Competencias a desarrollar en el programa de Ingeniería Mecánica

\section{Competencias Proyecto Educativo del Programa}

Durante su permanencia en el programa el estudiante adquirirá destrezas y aptitudes para:

1. Formular y solucionar problemas fundamentales en el campo de las matemáticas.

2. Resolver problemas que demanden el razonamiento abstracto y lógico, la inducción y deducción.

3. Comprender, contextualizar, interpretar y adaptar textos y artículos pertinentes al escenario 


\begin{tabular}{|c|l|}
\hline tecnológico. \\
\hline 4. & $\begin{array}{l}\text { Tener una visión futurista para la construcción de su } \\
\text { proyecto de vida. }\end{array}$ \\
\hline 5. & $\begin{array}{l}\text { Tener un compromiso con el entorno social y } \\
\text { regional. }\end{array}$ \\
\hline 6. & $\begin{array}{l}\text { Establecer buenas relaciones interpersonales y de } \\
\text { trabajo en equipo. }\end{array}$ \\
\hline 7. & $\begin{array}{l}\text { Mantenerse en constante actualización de sus } \\
\text { conocimientos y destrezas }\end{array}$ \\
\hline 8. & Tener una capacidad auto critica. \\
\hline
\end{tabular}

Se evidencia en el tipo de competencia número cinco, en donde se plantea para los estudiantes de ingeniería mecánica elementos de competencias del saber ser en donde se propone que dentro de su formación profesional se encaminen acciones de enseñanza - aprendizaje orientado al compromiso con el entorno social y regional. Este elemento, permite que al desarrollar las diferentes asignaturas, desde su modelo de micro currículo se propongan proyectos de aula o de investigación formativa en áreas de la protección del medio ambiente, con soluciones sustentables para el sector productivo de la región.

Se presenta a continuación los resultados del instrumento aplicado a estudiantes y docentes del programa de Ingeniería Mecánica matriculados durante el segundo semestre de 2016.

En primera estancia a los estudiantes que fueron encuestados cursan entre el tercer y octavo semestre del programa de Ingeniería Mecánica. La muestra de 218 estudiantes fueron los que se encontraban matriculados en asignaturas tales como: Electromagnetismo, Procesos de Manufactura, Ingeniería Económica, Estática, Ciencia de los Materiales, Mecánica de Fluidos, Cálculo Vectorial, Dibujo de Máquinas con el fin de realizar un diagnóstico para el programa y se logren evidenciar las áreas de mejora.

Para diagnosticar el área de mejora del Sistema de Gestión Ambiental (SIGA) y su inserción con el Programa de Ingeniería Mecánica de la Universidad Francisco de Paula Santander Ocaña, es momento de realizar a partir del diagnóstico participativo con docentes y estudiantes establecer las fortalezas, debilidades, puntos críticos y finalmente demandas y sugerencias para que se asuman como un compromiso por parte de los lineamientos del plan de estudios para llevarlo a acciones de mejora específicas aprobadas por el comité curricular del plan de estudios. Se describe a continuación una matriz de mejora que permite sean identificadas las áreas del plan de estudios en las cuales serán implementadas las acciones de mejora.

La tabla 2 relaciona los resultados encontrados una vez aplicado el instrumento de medición, en cada una de sus preguntas:

Tabla 2: Percepción de los estudiantes del programa

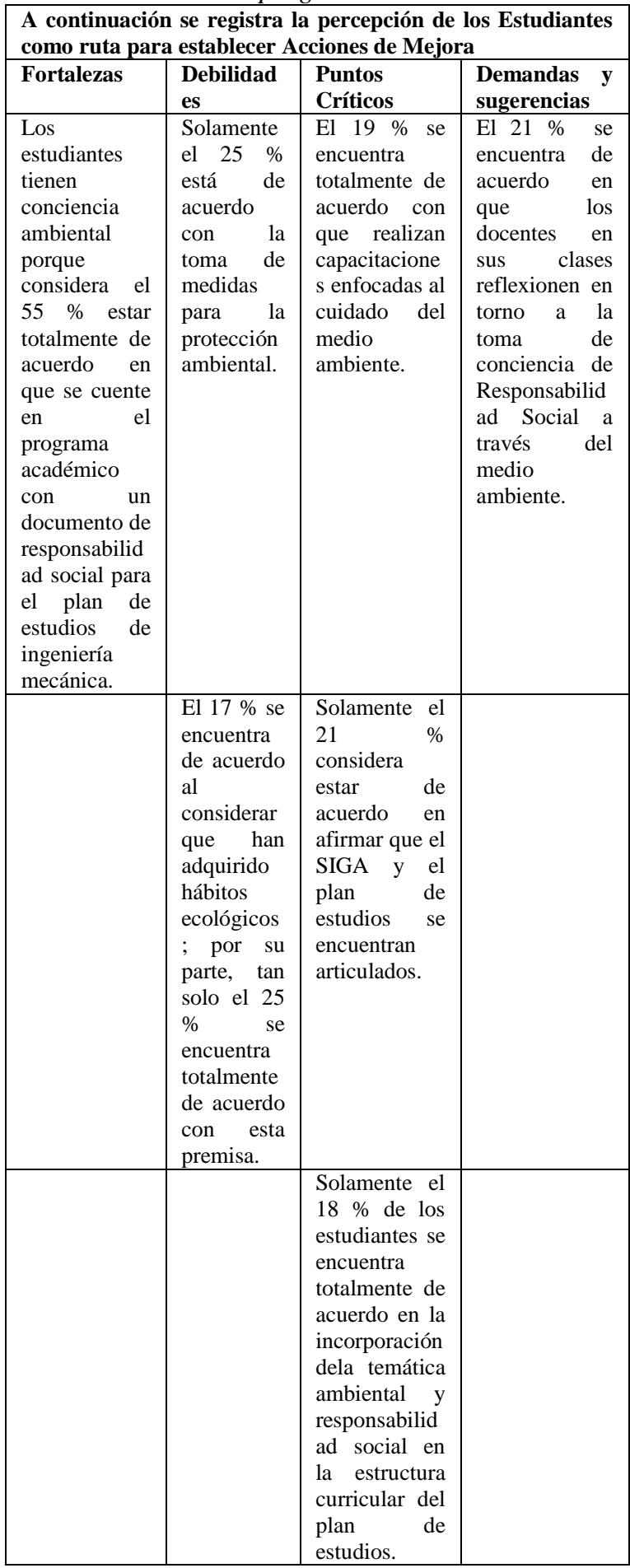


Se describe a continuación la matriz de acciones de mejora que corresponde a los docentes:

Tabla 3. Percepción de los docentes del programa

\begin{tabular}{|c|c|c|c|}
\hline \multicolumn{4}{|c|}{$\begin{array}{l}\text { A continuación se registra la percepción de los docentes } \\
\text { como ruta para establecer Acciones de Mejora }\end{array}$} \\
\hline Fortalezas & $\begin{array}{l}\text { Debilidad } \\
\text { es }\end{array}$ & $\begin{array}{l}\text { Puntos } \\
\text { Críticos }\end{array}$ & $\begin{array}{l}\text { Demandas } y \\
\text { sugerencias }\end{array}$ \\
\hline $\begin{array}{l}\text { Los docentes } \\
\text { del programa } \\
\text { de Ingeniería } \\
\text { Mecánica de } \\
\text { la } \\
\text { Universidad } \\
\text { Francisco de } \\
\text { Paula } \\
\text { Santander } \\
\text { Ocaña } \\
\text { consideran en } \\
\text { un } 40 \% \text { que } \\
\text { la institución } \\
\text { es } \\
\text { ambientalme } \\
\text { nte } \\
\text { responsable. }\end{array}$ & $\begin{array}{l}\text { Solamente } \\
\text { el } 10 \% \text { se } \\
\text { encuentran } \\
\text { totalmente } \\
\text { de acuerdo } \\
\text { al } \\
\text { considera } \\
\text { que el } \\
\text { personal } \\
\text { docente y } \\
\text { no docente } \\
\text { recibe } \\
\text { capacitació } \\
\text { n en } \\
\text { temáticas } \\
\text { de } \\
\text { preservaci } \\
\text { ón y } \\
\text { cuidado } \\
\text { del medio } \\
\text { ambiente. }\end{array}$ & $\begin{array}{l}\text { Tan solo el } 20 \\
\% \text { considera } \\
\text { que se tiene } \\
\text { coherencia } \\
\text { entre los } \\
\text { principios y la } \\
\text { política } \\
\text { ambiental de } \\
\text { la institución } \\
\text { educativa } \\
\text { UFPS Ocaña. }\end{array}$ & $\begin{array}{l}\text { El } 80 \% \text { de los } \\
\text { docentes } \\
\text { consideran } \\
\text { estar } \\
\text { totalmente de } \\
\text { acuerdo en } \\
\text { que la } \\
\text { estructura } \\
\text { curricular del } \\
\text { programa } \\
\text { debe contar } \\
\text { con la } \\
\text { inclusión de } \\
\text { temáticas de } \\
\text { cuidado del } \\
\text { medio } \\
\text { ambiente y } \\
\text { Responsabilid } \\
\text { ad Social } \\
\text { Universitaria. }\end{array}$ \\
\hline $\begin{array}{l}\text { El 50 \% se } \\
\text { encuentra } \\
\text { totalmente de } \\
\text { acuerdo en } \\
\text { que se tiene } \\
\text { la política } \\
\text { institucional } \\
\text { para la } \\
\text { protección } \\
\text { del medio } \\
\text { ambiente. }\end{array}$ & $\begin{array}{l}\text { Tan solo el } \\
20 \quad \% \\
\text { considera } \\
\text { estar de } \\
\text { acuerdo } \\
\text { con que se } \\
\text { tienen } \\
\text { hábitos } \\
\text { ecológicos } \\
\text {. }\end{array}$ & $\begin{array}{l}\text { El } 40 \% \text { se } \\
\text { encuentra } \\
\text { totalmente de } \\
\text { acuerdo en } \\
\text { que se debe } \\
\text { contar con un } \\
\text { documento } \\
\text { que defina la } \\
\text { política de } \\
\text { Responsabilid } \\
\text { ad Social para } \\
\text { el programa } \\
\text { de mecánica. }\end{array}$ & \\
\hline
\end{tabular}

Así como lo plantea Hernández \& Saldarriaga (2008) el papel que juega la Universidad como agente de transformación de sociedad al entregar un valor humanizante al estudiante que ha recibido una formación integral. Es así, como el programa de Ingeniería Mecánica entrega a la región un ser que busca transformar y ser solucionador de problemas en su entorno; además, forma profesionales que sean capaces de poner sus conocimientos y competencias al servicio de la comunidad y la industria, a través de la orientación de las asignaturas del pensum hacia las necesidades actuales del sector productivo y la comunidad en la región. Es una labor del comité curricular del programa encaminar el desarrollo de las materias que conforman el pensum para que exista confrontación directa de las situaciones que suceden en el entorno empresarial y social con los análisis y aproximaciones que se realizan en la academia con un enfoque al uso eficiente de la energía y cuidado del medio ambiente(Herrera, 2008).

Se propone para la inclusión del Sistema de Gestión Ambiental en el programa de Ingeniería Mecánica, de acuerdo a lo establecido en el instructivo de elaboración de documentos en el Sistema Integrado de Gestión en su ítem 6.4 se establece para la elaboración de manuales o guías que se cuente con la información específica, instrucciones o acuerdos necesarios para la ejecución del trabajo asignado con el fin de cumplir disposiciones reglamentarias; para lograr como resultado el siguiente contenido: presentación, introducción, contenido del documento, información de elaboración, revisión y aprobación, control de cambios.

La Universidad Francisco de Paula Santander Ocaña a través del proyecto educativo de la Facultad " promueve, como ente generador y de formación en ámbitos científicos y tecnológicos, la docencia, la investigación y la extensión, como pilares fundamentales del desarrollo pedagógico y social, trazando lineamientos desde la ingeniería, que incidan en el cambio regional y nacional"(Sánchez Ortiz, 2012), de esta manera, se integran varios procesos, en los cuales la capacitación docente en el uso y apropiación pedagógica de las Tecnologías de la Información y la Comunicación (TIC) se convierte en una herramienta importante que logra fortalecer en docentes y estudiantes las competencias genéricas y específicas durante el proceso de formación del programa de Ingeniería Mecánica (Franky, 2012).

El manual propuesto es una guía para establecer la planificación y operación del SIGA en la formulación de las acciones que pueden contribuir con la protección ambiental y la prevención de la contaminación en el programa de ingeniería mecánica adscrito a la Facultad de Ingenierías de la U F P S Ocaña, cuya implementación se ajusta a los requisitos de la norma N T C ISO 14001: 2004 y en los estándares y modelos como el MECI y Autoevaluación con enfoque hacia la alta acreditación de acuerdo a los lineamientos del Consejo Nacional de Acreditación (CNA)(Gómez Luna et al., 2016).

A partir de los procedimientos, planes y programas del Sistema de Gestión Ambiental (SIGA) se establecen aquellos que aplican para este plan de estudios como objeto de estudio en la presente investigación. A continuación se registra en la tabla 4, la identificación de los usuarios (ICONTEC, 2015). 
Se hace referencia entonces al compromiso con el entorno y para garantizar su cumplimiento se considera en la política integral de la institución un eje ambiental que propone se incorpore la ética ambiental en las actividades de docencia e investigación. Es así como las acciones en los programas académicos se orientan a dar cumplimiento a los siguientes compromisos (Sanchez Ortiz, 2015):

\section{Tabla 4: Cumplimiento de la Política Ambiental de la organización}

\begin{tabular}{|c|c|c|c|}
\hline Compromiso & $\begin{array}{l}\text { Acciones } \\
\text { UFPSO }\end{array}$ & $\begin{array}{l}\text { Acciones de } \\
\text { cumplimiento } \\
\text { en el programa } \\
\text { de ingeniería } \\
\text { Mecánica }\end{array}$ & $\begin{array}{l}\text { Responsa } \\
\text { ble }\end{array}$ \\
\hline $\begin{array}{l}\text { Disminución } \\
\text { del impacto } \\
\text { ambiental, } \\
\text { optimización } \\
\text { y eficiencia } \\
\text { operacional }\end{array}$ & $\begin{array}{l}\text { Disposición } \\
\text { adecuada de } \\
\text { residuos } \\
\text { comunes }\end{array}$ & $\begin{array}{l}\text { Aplicación del } \\
\text { procedimiento } \\
\text { de gestión de } \\
\text { los residuos } \\
\text { sólidos y } \\
\text { peligrosos en } \\
\text { cada uno de } \\
\text { los Laboratorio } \\
\text { adscritos al } \\
\text { departamento } \\
\text { de Ingenierías } \\
\text { Mecánica. } \\
\text { Máquinas y } \\
\text { Herramientas }\end{array}$ & $\begin{array}{l}\text { Coordinador } \\
\text { de } \\
\text { laboratorio } \\
\text { adscritos al } \\
\text { programa } \\
\text { académico: } \\
\text { Laboratorio } \\
\text { de Máquinas } \\
\text { y } \\
\text { Herramientas } \\
\text { Laboratorio } \\
\text { Maquinas } \\
\text { térmicas y } \\
\text { transferencia } \\
\text { de calor. } \\
\text { Laboratorio } \\
\text { Mecánica de } \\
\text { Fluidos } \\
\text { Laboratorio } \\
\text { Metalografía. } \\
\text { Docente } \\
\text { Director de } \\
\text { Proyecto }\end{array}$ \\
\hline $\begin{array}{l}\text { Cumplir } \\
\text { requisitos } \\
\text { legales }\end{array}$ & $\begin{array}{l}\text { Manejo } \\
\text { ambiental } \\
\text { integral en la } \\
\text { generación de } \\
\text { vertimientos } \\
\text { Implementació } \\
\text { n de controles }\end{array}$ & $\begin{array}{l}\text { Revisión de } \\
\text { acciones en el } \\
\text { Modelo de } \\
\text { Micro currículo } \\
\text { en asignaturas } \\
\text { que hacen parte } \\
\text { de las líneas } \\
\text { del programa. } \\
\text { Revisión de las } \\
\text { acciones } \\
\text { operativas para } \\
\text { el } \\
\text { funcionamiento } \\
\text { del plan de } \\
\text { estudios de } \\
\text { ingeniería } \\
\text { Mecánica. } \\
\text { Acciones de } \\
\text { seguimiento de } \\
\text { los practicantes } \\
\text { y/o pasantes } \\
\text { con el sector }\end{array}$ & $\begin{array}{l}\text { Secretaria } \\
\text { del } \\
\text { programa } \\
\text { y/o } \\
\text { administrativ } \\
\text { os }\end{array}$ \\
\hline
\end{tabular}

productivo.

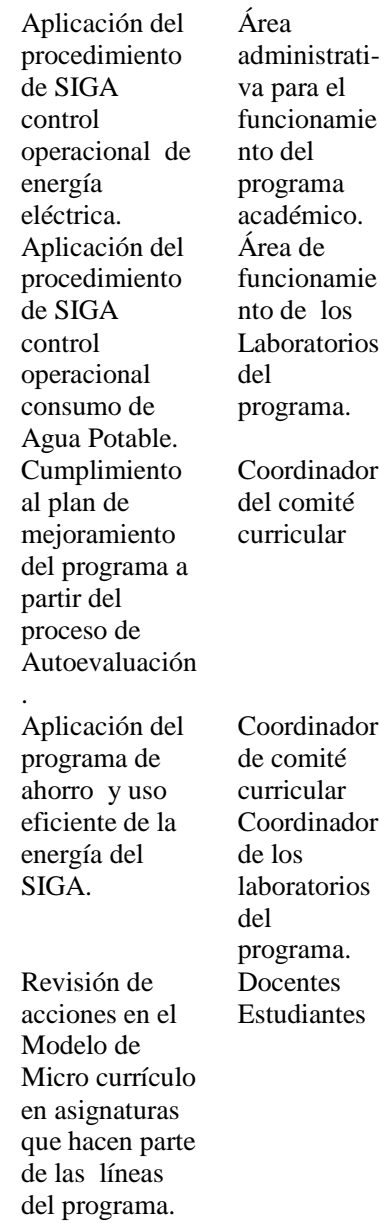

\section{CONCLUSIONES}

Para docentes y estudiantes es importante que el plan de estudios viabilice acciones en torno a la protección y preservación del medio ambiente; con el fin de formar ingenieros mecánico integrales con responsabilidad social siendo un solucionador de problemas en medio de una sociedad que requiere profesionales idóneos que desarrollen sus funciones con propósitos sostenibles y teniendo en cuenta la protección y preservación del medio ambiente. A partir del análisis realizado en el referente teórico se logra evidenciar para la caracterización del programa de Ingeniería Mecánica de la Universidad Francisco de Paula Santander Ocaña, a través de la misión del plan de estudios se define el hombre que se quiere formar de acuerdo a lo establecido en el Proyecto Educativo y objetivos del Programa, que se caracteriza por ser propositivo y solucionador de problemas en medio de una sociedad que requiere profesionales con compromiso social. Se cuenta 
con un enfoque pedagógico constructivista social, que permite identificar elementos didácticos que se implementan en el aula de clase a través del modelo de micro currículo. Con ello se tiene identificado en el programa académico asignaturas, proyectos de aula y pasantías que garantizan el compromiso social del programa académico.

\section{REFERENCIAS}

Bonfante, M. C., \& Castillo, A. (2014). MULTIAGENT SYSTEM, ONTOLOGIES AND BUSINESS PROCCESSES INTEGRATION AS TECHNOLOGY FRAMEWORK FOR "THE GOVERNMENT ONLINE" STRATEGY. Revista Colombiana de Tecnologías de Avanzada.

Espinel Blanco, E. E. (2012). Documento Maestro PEP Ingenieria Mecanica 2012.

Franky, G. A. (2012). PEDAGOGICAL MASTERING OF NEW INFORMATION AND COMMUNICATION TECHNOLOGIES (NICTS). TEACHER TRAINING EXPERIENCE. Revista Colombiana de Tecnologías de Avanzada, 2(20) pp. 57-64.

G, A. A. Á., Serrano, E. G. F., \& G., O. G. (2012). DESIGN AND IMPLEMENTATION OF A MULTIMEDIA INTERACTIVE COURSE FOR THE LEARNING OF PROCESSES IN CAM A MACHINING CENTER LEADWELL V30. Revista Colombiana de Tecnologías de Avanzada.

Gomez Luna, L., Franco Rodríguez, M., Muñoz Martínez, S., Reynel Chila, V., Serrano Guerrero, S., Andrade Benalcázar, D., ... Ayoví, N. (2016). Bases para el tránsito a la Excelencia en universidades públicas del Ecuador: Actividades clave y propuesta de indicadores para la Universidad Luis Vargas Torres, de Esmeraldas. Revista Científica Interdisciplinaria Investigación Y Saberes, V, 68-93.

Hernandez, R., \& Saldarriga, A. (2009). Gestión de la responsabilidad social universitaria. Caso: Escuela de Ingeniería de Antioquia -EIA-. Dyna, (159), 237-248.

Herrera, A. (2008). La responsabilidad social universitaria en América Latina. Revistes I Congressos UPC, 17, 283-304. Retrieved from http://upcommons.upc.edu//handle/2099/797 9

ICONTEC. (2015). NTC ISO-9001:2015. Iso,
9001, 33. https://doi.org/ISBN 978-92-67$10650-2$

Mesa, L., \& Barrera, N. (2012). La Robótica Educativa Como Instrumento Didáctico Alternativo En Educación Básica. Revista Colombiana de Tenologia de Avanzada, 96103.

Puerto Suarez, J. D., \& Lopez Jimenez, V. L. (2014). Responsabilidad Social Universitaria, Gestión del Riesgo y Resilencia Institucional. Visión de la Universidad de la Salle. In Educación, pedagogía y sociedad (p. 35).

Rodríguez, O. O., Pinto, R. F. P., \& Cárdenas, P. F. (2012). Lineal System To the Inverted Pendulum on Car Slide. Revista Colombiana De Tecnologias De Avanzada (Rcta), 1(19). https://doi.org/10.24054/01204211.V19.N19. 2012.153

Sanchez Ortiz, E. A. (2012). Proyecto Educativo de la Institución (PEI). Ocaña. Retrieved from https://ufpso.edu.co/ftp/pdf/documentos/PEI5 .pdf

Sanchez Ortiz, E. A. (2015). MANUAL DEL SISTEMA.

Vallaeys, F., De La cruz, C., \& Sasia, P. (2009). Manual para la responsabilidad Social Universitaria. Manual de primeros pasos. Journal of Chemical Information and Modeling (Vol. 53). https://doi.org/10.1017/CBO9781107415324. 004 